\title{
The Formulation and Discretization Analysis Using the BEM
}

\author{
Twaibu Semwogerere ${ }^{1}$
}

\begin{abstract}
Boundary discretization has been has been an ideal technique in solving boundary problems that are a common phenomenon in mathematics. The boundary element method (BEM) is one of the methods which has had an advantage of discretizing the boundary than the finite element method (FEM) and the finite difference method FDM) for quite so long. The curved and straight elements are crucial for the discretization process. In this study, the BEM has been used to analyze and compare curved or straight boundary discretization.
\end{abstract}

The BEM and its applications was reviewed in relation to curved and straight elements. The Laplace equation with its boundary integral formulation was done and the BEM applied. The Dirac-Delta and the Green's functions were behind the use of the BEM. Finally, three model problems were tested for analysis of the BEM with curved and straight elements in relation to solving the Laplace problem. The MATLAB programs and subprograms were used among others to solve the problem and also used in the analysis.

Findings showed the fundamental advantageous stage of the curved to straight elements, the easy formulation of the latter to the former, and the suitability of the former to the latter when considering the curved and straight boundaries respectively.

Keywords - Boundary, Curved, Straight, Elements, Formulation.

$1 \quad$ Ph. D Student, College of Engineering, Design, Art and Technology (CEDAT), Makerere University, P. O. Box, 7062, Kampala Uganda.

\section{INTRODUCTION}

Several mathematical problems arise in various daily life encounters. Some of these problems are the boundary problems [1] [2]. Such problems can be solved by several analytical and numerical methods which include the BEM, among others. The BEM simply requires the discretization of the boundary than the whole domain. It should be noted that the BEM is more recent than the other two and has several advantages over the others that include among others: Discretizing at infinity [3], and its application to solving acoustic and general engineering science problems [2].

This manuscript mainly entailed the formulation of the straight and curved elements for integral formulation, and the use of the BEM on these elements. The Dirac-delta distributions, the Green's functions, the Laplace equation, the BEM and the divergence theorem were necessarily reviewed.

\subsection{The Laplace equation}

The equation is used in electro-statistics, incompressible fluid flow systems and for the heat conduction in the steady state [2], [4]. For a Laplace equation, the solutions within a given region say $R$, can be found given the specification of the potential or field around the boundary. Solutions of Laplace's equation are called harmonic functions [5]. The Laplace's boundary integral form required us to transform the PDE only unlike the FEM. A function $\boldsymbol{u}$ was considered to be satisfying the equation to give the following integral formulations:

$$
\begin{aligned}
& \iint_{D}\left[G(x ; \xi) \nabla^{2} u(x(S))-u(x) \nabla^{2} G(x ; \xi)\right] d A=\iint_{D}\left[-u(x) \nabla^{2} G(x ; \xi)\right] d A \\
& \int_{S}\left[G(s ; x) \frac{\partial u}{\partial n}(s)-u(s) \frac{\partial G}{\partial n}(s)\right] d s=\left\{\begin{array}{c}
u(x), \xi \in D \\
0.5 u(x), \xi \in S \\
0, \xi \in E
\end{array}\right.
\end{aligned}
$$

Where $G$ is the Green's function, $D$ is the interior domain, $S$ is the boundary and $E$ is the exterior region. Equation (1) is simplified by applying Green's function properties, and by cosmetic changes. This accomplishes the first stage of the BEM [1], [2].

\subsection{The Boundary Element Method (BEM)}

\subsubsection{Introduction}

The BEM as applied to the Laplace's equation is the most recent than the FEM and FDM [2], [6] and [7]. In the last three decades, the method has been developed into a robust technique for especially modeling elasticity and acoustics [2], [8] and [9]. The term "element" meant the geometry and type of approximation to a given variable. The term "node" was used to define the element geometry or the variables involved in the problem.

From [2], we noted that in using the BEM on the interior Laplace problem, two relevant things were noted about elements;

The different number of nodes were used for the variable approximations; and the approximation being be linear, quadratic or otherwise. But some of these approximations like the linear and quadratic use global basis functions which are continuous over the boundary.

It was also noted from [2] that the change of the

derivative $\frac{\partial u}{\partial n}=v$ at these nodes may be difficult to overcome. It is advantageous however to use the interior nodes only as with the constant or Guass-Legendre points especially when the geometry is not smooth.

We sometimes use straight line segments to represent the geometry, and the mid-points of such elements to represent the nodes. We also let the global approximations to be:

$u(s)=\sum_{c=1}^{n} \varphi_{c}(s) \boldsymbol{u}_{c}, \quad v(s)=\sum_{c=1}^{n} \varphi_{c}(s) \nu_{c}$

Where $\varphi_{c}(s)$, are the boundary element basis functions, and $\mathrm{n}$ represents the nodes number.

Now, S, the actual boundary was replaced by $\bar{S}$ (union of straight elements). Hence by applying the variable approximation and considering the BEM for the exterior form, equation (1) was simplified to: 


$$
\left.\sum_{c=1}^{n}\left[\underset{s_{c}}{[} G(x ; s) d s\right] \mathcal{V}_{c}-\left[\int_{-c} \frac{\partial G}{\partial n}(x ; s) d s\right] \boldsymbol{U}_{c}\right]=\left\{\begin{array}{c}
-u(x) \text { if } x \in D \\
-0.5 u(x) \text { if } x \in S \\
0 \text { if } x \in E
\end{array}\right.
$$

These variables were assumed to be constant on each element. For this case we chose one node per element, hence the same number of nodes and elements. As earlier noted, two steps are used in the method: Obtaining $\underline{u}$ and $\underline{v}$ or using $\underline{u}$ and $\underline{v}$ to obtain $\boldsymbol{u}$ at some general point. For the former, the collocation method was applied. It is at the collocation point that the relation between $\underline{u}$ and $\underline{v}$ is forced to hold. With this method the variable point $\mathrm{x}$ is brought to each of the boundary nodes in turn. The point $\boldsymbol{q}_{r}$ was let to be the mid-point of element $r$. This led to the following equivalent equations [2]:

$$
\begin{gathered}
\sum_{c=1}^{n}\left[\left[\int_{S^{c}} G\left(q_{r} ; s\right) d s\right] \boldsymbol{v}_{c}-\left[\int_{s} \frac{\partial G}{\partial n}\left(q_{r} ; s\right) d s\right] \boldsymbol{u}_{c}\right]=0.5 \boldsymbol{u}_{r} \\
\sum_{c=1}^{n}\left[\boldsymbol{L}_{r c} \boldsymbol{v}_{c}-\boldsymbol{M}_{r c} \boldsymbol{u}_{c}\right]=0.5 \boldsymbol{u}_{r}, \boldsymbol{L}_{r} v-\boldsymbol{M}_{r} \boldsymbol{u}=\mathbf{0 . 5 \boldsymbol { u } _ { r }}
\end{gathered}
$$

Where $\mathrm{r}=1 \ldots \mathrm{n}$, and $\mathrm{n}$ is the number of nodes. The exterior formulation simply negates the RHS of equation (4).

It was noted that equation (4) could be written in matrix form as $L \underline{v}-(M-0.5) \underline{u}=0$ for the exterior case [6]. For a Dirichlet problem where $\underline{u}$ is given, $\underline{v}$ can be found. We also noted on the other hand if it is a mixed problem, the resulting matrices have to be ordered and re-partitioned. The matrices $\boldsymbol{L}_{r c}$ and $\boldsymbol{M}_{r c}$ were resolved using the equations below for both the straight and curved elements.

$$
L_{r c}=\int_{\bar{S}} G\left(q_{r} ; s\right) d s \text { and } \mathbf{M}_{\mathrm{rc}}=\int_{\bar{S}} \frac{\partial G}{\partial n}\left(q_{r} ; s\right) d s
$$

To resolve these integrals, we assumed the availability of the coordinates of the collocation point $\boldsymbol{q}_{r}$ and the end points a, b. The diagonal entries arise when $\boldsymbol{q}_{r}$ coincides with the element node. This accomplished the first step, because at this stage $\underline{v}$ could be calculated for a Dirichlet problem [6]. In the second step $\underline{u}$ at a general point could be found by

$$
u(x)=M(x) \underline{u}-L(x) \underline{v}
$$

Resolving the matrix and integral forms for the straight and curved elements can be found in the findings of this manuscript.

\section{METHODOLOGY}

The BEM, Dirac-Delta and the Green's functions were also briefly reviewed in relation to the Laplace problem in the finite space. The Laplace equation with its boundary integral formulation was done and the BEM applied. The algebra behind the straight and curved elements was done followed by three model problems that were tested for analysis. Several MATLAB programs were used in the analysis. They included among others:

Beintc.m: This uses the BEM for solving the Laplace's equation in the interior of the unit circle as applied to curved elements for a
Dirichlet type problem. The element data (discretization), is done by the file Elts.m while the $\underline{u}$ data is calculated from the exact solution by using the sub-program Mant2.m. This function Mant2.m computes vectors $L_{r}, M_{r}$ which are the rows of the boundary element matrices $L$ and $M$. The $L_{x}, M_{x}$ vectors in the second stage of the BEM also use this function. It also uses a function called Trapez.m to solve quadratic-in-sine integrals (Semo, 2015).

Beint.m: This uses the BEM for solving the Laplace's equation in the interior of the unit square as applied to Straight elements for a Dirichlet type problem. This did not require sub-programs but the actual programming of the sub-functions. The formulation analysis details were not done in this manuscript since they are found in [2].

\section{FINDINGS}

\subsection{Computation of the matrix terms for straight elements}

The straight element considered was represented as a straight line from A to B. In this section, we show how the entries for the matrices $\mathrm{M}$ and $\mathrm{L}$ are computed with respect to straight elements. The integrals for $\mathrm{M}$ and $\mathrm{L}$ are evaluated by mapping the straight element $\mathrm{AB}$ on to $[-1,1]$ by means of

$$
S=\frac{(1+t) l c}{2}{ }_{\text {so that }} d s=\frac{l c}{2} d t
$$

Fig. 1 below illustrates the setting that was used to compute the matrix terms for straight elements.

From Fig. 1 and the mapping above,

$$
\begin{gathered}
q_{c}=0.5(a+b) ; ;^{q(t)=q_{c}+t d} l^{c}=2 \sqrt{d . d} ; c=q_{c}-q_{r} \\
n=2[d(2), d(1)] / l^{c} ; r(t)=q(t)-q_{r}=t d+c
\end{gathered}
$$

Hence,

$$
r . n=c . n \text { and } r^{2}=r . r=\alpha t^{2}+2 \beta t+\gamma
$$

Where $\alpha=d . d, \beta=c . d, \gamma=c . c$

Therefore,

$$
M_{r c}=\int_{\overline{s_{c}}} \frac{\partial G}{\partial n}\left(q_{r} ; s\right) d s
$$

$$
\begin{aligned}
=-\int_{-1}^{1} \frac{(r . \underline{n}) l^{c} d t}{4 \pi r^{2}} \\
L_{r c}=\int_{s_{c}} G\left(q_{r} ; s\right) d s \\
\quad=-\int_{-1}^{1} \frac{l^{c} \ln r d t}{4 \pi}
\end{aligned}
$$

In order to resolve the integrals in (10) and (11), we assumed the availability of the coordinates of the collocation point $q_{r}$, and the end points $a$ and $b$. The diagonal entries arise when the collocation point coincides with the element node. These integrals have singularities but are still integrable.

$$
M_{r r}=0 \text { and } L_{r r}=\frac{l^{r}}{2 \pi}\left[1-\ln \left(\frac{l^{r}}{2}\right)\right] \text {, for } r=1,2, \ldots, n \text {. }
$$




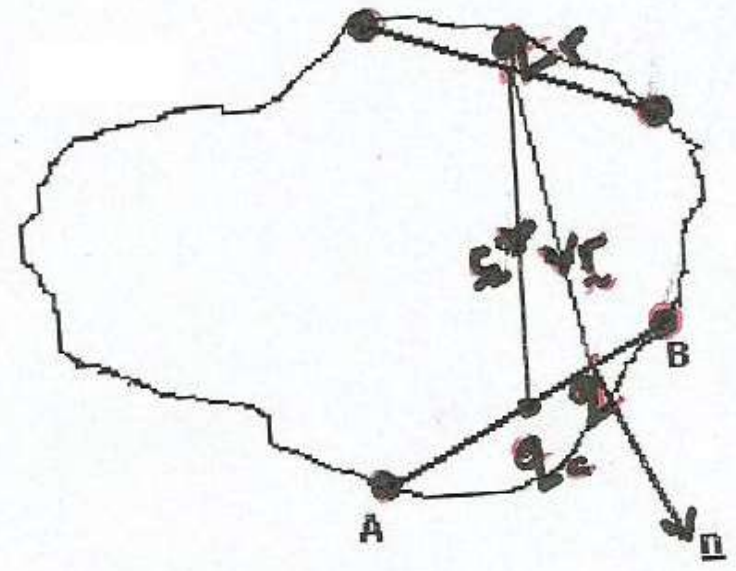

Figure. 1. The Setting for Computing the Matrix Terms for Straight Elements

\subsection{Computation of the matrix terms for curved elements}

Some element-discretization procedures and variable approximation techniques like those above remained the same as for the straight elements. We therefore began the computation of the terms for the matrices $\boldsymbol{L}_{r c}$ and $\boldsymbol{M}_{r c}$ already considered in the previous section. A curved boundary element, as an arc of a circle, $\mathrm{AB}[2]$.

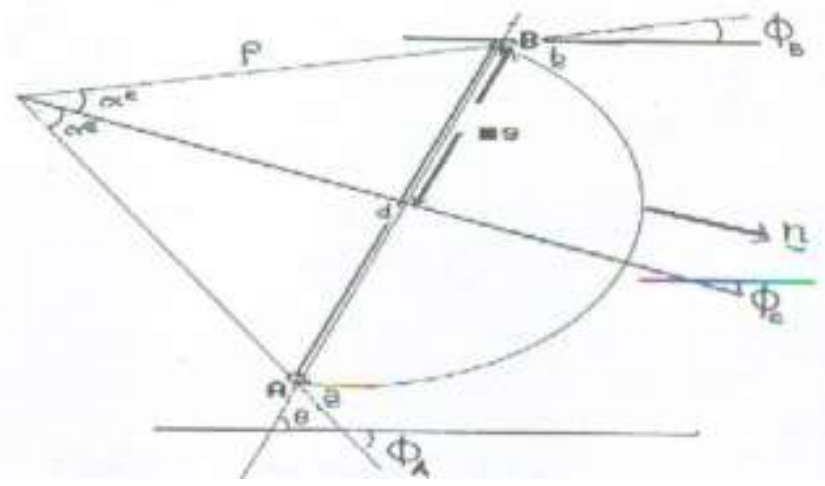

Figure 2. The Setting for Computing the Matrix Terms for Curved Element [2].

From Figure.2, there are various expressions that were resolved as shown in [2]. This manuscript simply entailed some few details about the curved elements. The integrals $\mathrm{M}$ and $\mathrm{L}$ were evaluated by mapping the curved element on to $[-1,1]$ as seen in [2].

$$
\begin{gathered}
M_{r c}=-\frac{l_{c}}{4 \pi} \int_{-1}^{1} \frac{(\underline{c}+\underline{p}) \cdot \underline{n} d t}{\lambda \sin ^{2}\left(\frac{\left.\alpha^{e} t\right)}{2}\right)+\cos \left(\frac{\left.\alpha^{e} t\right)}{2}\right)+\gamma} \\
L_{r c}=-\frac{l_{c}}{8 \pi} \int_{-1}^{1} \ln \left(\lambda \sin ^{2}\left(\frac{\left.\alpha^{e} t\right)}{2}\right)+\cos \left(\frac{\left.\alpha^{e} t\right)}{2}\right)+\gamma\right)
\end{gathered}
$$

The integrals in equations (13) were elevated using the trapezoidal rule with a varying number of ordinates. However the diagonal elements given by the integrals $\boldsymbol{M}_{r r}$ and $\boldsymbol{L}_{r r}$ required a different approach because a singularity cannot be avoided. In order to simplify the singularity problem, we resolved that [2], [9]:

$$
\begin{gathered}
M_{r r} \text { is zero and when } \boldsymbol{q}_{c}=q_{r} r(t)=\underline{p}=2 \rho \sin \left(\frac{\left.\alpha^{e} t\right)}{2}\right) \\
\frac{-l_{c}}{4 \pi} \int_{-1}^{1} \ln \left(\frac{\alpha^{e} t}{2}\right) d t=\frac{-l_{c}}{4 \pi}\left(\ln \left(\frac{l_{r}}{2}\right)-2\right) r=1,2, \ldots, n . \\
r=c \text { and } \rho \alpha^{e}=\frac{l_{c}}{2}
\end{gathered}
$$

\subsection{Model Problems}

\subsubsection{Problem 1}

It was required to recover the solution of

$$
u(x, y)=\sin \pi x \sinh \pi y
$$

at $(0.5,0.5)$ on the unit square, with Dirichlet conditions taken from the solution.

At the four nodes, the following $u(x, y)$ was obtained:

$$
\boldsymbol{u}=\left[\begin{array}{llll}
0 & 0 & 1 & 1.5487
\end{array}\right]
$$

The computation of the matrix terms was easy. Only two entries were evaluated as a sample:

$$
\begin{gathered}
M_{13}=-l_{3} \frac{c . n}{4 \pi} \int_{-1}^{1} \frac{d t}{\alpha t^{2}+2 \beta t+\gamma} \\
=-\frac{1}{4} \int_{-1}^{1} \frac{d t}{t^{2}+4} \\
L_{13}=\frac{l_{3}}{4 \pi} \int_{-1}^{1} \ln r d t=-0.0062
\end{gathered}
$$

Therefore, the entries of the matrices $M$ and $L$ were finally given as:

$$
\begin{aligned}
& M=\left(\begin{array}{cccc}
0.0000 & -0.1762 & -0.1476 & 0.1762 \\
-0.1762 & 0.0000 & 0.1762 & -0.1476 \\
-0.1476 & -0.1762 & 0.0000 & -0.1762 \\
-0.1762 & -0.1476 & -0.1762 & 0.0000
\end{array}\right) \\
& L=\left(\begin{array}{cccc}
0.2695 & 0.0533 & -0.0062 & 0.0533 \\
0.0533 & 0.2695 & 0.0533 & -0.0062 \\
-0.0062 & 0.0533 & 0.2695 & 0.0533 \\
0.0533 & -0.0062 & 0.0533 & -0.0062
\end{array}\right) \\
& \text { Then } \quad v=\left[L^{-1}(M+0.5 I)\right] u
\end{aligned}
$$

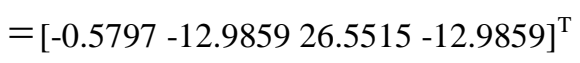

$$
\begin{aligned}
& \text { But also, } \quad v=\frac{\partial u}{\partial n_{c}}
\end{aligned}
$$

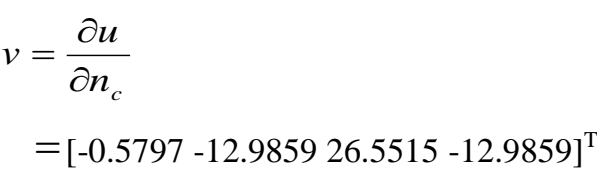$$
L(x)=\left[\begin{array}{llll}
0.0893 & 0.0893 & 0.0893 & 0.0893
\end{array}\right] \text { and }
$$$$
M(x)=[-0.2500-0.2500-0.2500-0.2500]
$$

Thus,

$$
u(0.5,0.5)=2.8872
$$


By varying the number of elements on the unit square, the Matlab program Beint. $m$ gave the following error bounds:

TABLE I. THE ERROR BOUNDS AS N VARIES ON A UNIT SQUARE

\begin{tabular}{|l|l|l|l|l|}
\hline Nodes $(n)$ & 16 & 80 & 160 & 320 \\
\hline $\begin{array}{l}\text { Error } \\
\left\|\boldsymbol{e}^{\infty}\right\|\end{array}$ & $0.4400 * 10^{0}$ & $0.3830 * 10^{-1}$ & $0.8070 * 10^{-3}$ & $870 * 10^{-3}$ \\
\hline
\end{tabular}

\subsubsection{Problem 2}

The solution of $\boldsymbol{u}(x, y)$ in equation (15) was required at $(0,0)$ on a unit circle, where the Dirichlet conditions are excluded in the solution[2]. At the four nodes,

$\underline{u}=\left[\begin{array}{llll}3.6252 & -3.6252 & 3.6252 & -3.6252\end{array}\right]^{\mathrm{T}}$. The singular integrals were solved by use of the trapezium rule and Gaussian quadrature. The following matrices show the evaluated entries:

$\begin{aligned} M & =\left(\begin{array}{cccc}-0.0000 & -0.0118 & -0.0053 & 0.0118 \\ -0.0118 & 0.0000 & 0.0118 & -0.0053 \\ -0.0053 & 0.0118 & 0.0000 & -0.0118 \\ 0.0118 & -0.0053 & -0.0118 & 0.0000\end{array}\right) \\ L & =\left(\begin{array}{cccc}0.2802 & -0.0632 & -0.1135 & -0.0632 \\ -0.0632 & 0.2802 & -0.0632 & -0.1135 \\ -0.1135 & -0.0632 & 0.2802 & -0.0632 \\ -0.0632 & -0.1135 & -0.0632 & 0.2802\end{array}\right)\end{aligned}$

$\underline{v}=\left[\begin{array}{llll}6.4137 & -6.4137 & 6.4137 & -6.4137\end{array}\right]^{\mathrm{T}}$ compared to the exact

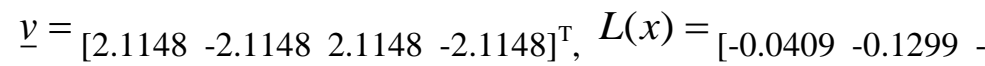
$\left.\begin{array}{ll}0.1223 & 0.2802\end{array}\right]^{\mathrm{T}}$ and $M(x)=\left[\begin{array}{llll}-0.0145 & -0.0043 & -0.0053 & 0.0000\end{array}\right]^{\mathrm{T}}$. Therefore $u(0,0)=-1.9545$ compared to the exact value $u(0,0)=0$. Table 2 gives the error bounds with varying nodes (element number) as seen below:

TABLE II. THE ERROR BOUNDS AS n VARIES ON A UNIT CIRCLE

\begin{tabular}{|c|c|c|c|c|c|}
\hline Nodes (n) & 8 & 16 & 32 & 64 & 128 \\
\hline Error $\left\|e^{\infty}\right\|$ & $\begin{array}{l}0.1097^{*} \\
10^{1}\end{array}$ & $\begin{array}{l}0.4323^{*} \\
10^{\circ}\end{array}$ & $\begin{array}{l}0.1286^{*} \\
10^{\circ}\end{array}$ & $\begin{array}{l}0.3820^{*} \\
10^{-1}\end{array}$ & $\begin{array}{l}0.1100^{*} \\
10^{-1}\end{array}$ \\
\hline
\end{tabular}

\subsubsection{Problem 3}

This problem looked at the convergence rates of either element types for the circle approximated by a polygon (straight elements) or exactly (curved elements). A circular boundary with known analytic solutions was considered. Figure 3 below shows the circular boundary and conditions considered. The Neumann conditions on the boundary were considered to be produced by a source point at $(0,1)$. Therefore

$$
V(\theta)=\frac{-(4-\cos \theta)}{2 \pi(17-8 \cos \theta)} ; 0 \leq \theta \leq 2 \pi
$$

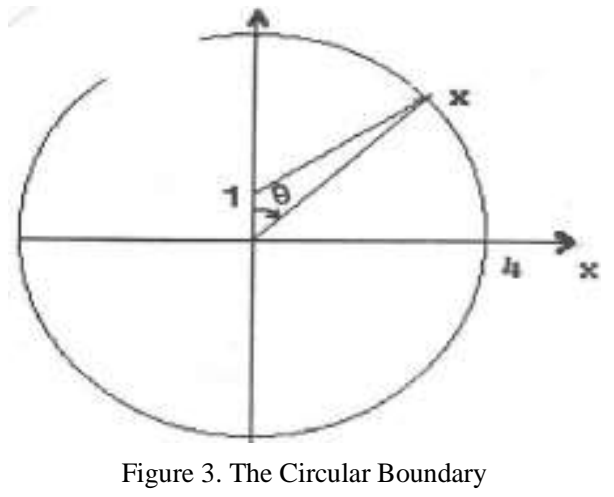

Equation (24) had the exact solution on the boundary given by

$$
u(\theta)=\frac{-\ln (17-8 \cos \theta)}{2 \pi}
$$

Where $\theta$ is the angle that $x$ subtends clockwise about the centre of the circle from the point $(0,4)$. The mean relative error (mre) to estimate the accuracy with respect to the direct methods only. This was given by:

$$
m r e=\frac{1}{n} \sum_{j=1}^{n} \frac{\left|\hat{u}_{j}-u_{j}\right|}{u_{j}}
$$

Where $u_{j}$ and $\hat{u}_{j}$ are respectively the computed and exact values of $u$ at the collocation points. The results for the different element types and numbers were displayed in the following tables [10].

TABLE III. mre FOR THE CONSTANT ELEMENTS ON THE CIRCLE APPROXIMATED EXACTLY

\begin{tabular}{|c|c|c|c|c|c|}
\hline Nodes $(n)$ & 8 & 16 & 32 & 64 & 128 \\
\hline mre & $\begin{array}{c}0.2260^{*} \\
10^{-4}\end{array}$ & $\begin{array}{c}0.7100^{*} \\
10^{-5}\end{array}$ & $\begin{array}{c}0.1930^{*} \\
10^{-5}\end{array}$ & $\begin{array}{c}0.5030^{*} \\
10^{-6}\end{array}$ & $\begin{array}{c}0.1280^{*} \\
10^{-6}\end{array}$ \\
\hline $\log _{2} \mathrm{mre}$ & - & - & - & - & - \\
& $0.8790^{*}$ & 0.1046 & 0.1234 & 0.1428 & 0.1625 \\
& $10^{1}$ & $* 10^{2}$ & $* 10^{2}$ & $* 10^{2}$ & $* 10^{2}$ \\
\hline
\end{tabular}

TABLE IV. mre FOR THE CONSTANT ELEMENTS ON THE CIRCLE APPROXIMATED BY A POLYGON

\begin{tabular}{|c|c|c|c|c|c|}
\hline Nodes $(n)$ & 8 & 16 & 32 & 64 & 128 \\
\hline Mre & $0.6970^{*}$ & $0.1800^{*}$ & $0.4550^{*}$ & $0.1150^{*}$ & $0.2870^{*}$ \\
& $10^{-3}$ & $10^{-3}$ & $10^{-4}$ & $10^{-4}$ & $10^{-5}$ \\
\hline $\log _{2} \mathrm{mre}$ & - & - & - & - & - \\
& $0.3840^{*}$ & $0.5800^{*}$ & $0.7780^{*}$ & $0.9770^{*}$ & $0.1177 *$ \\
& $10^{1}$ & $10^{1}$ & $10^{1}$ & $10^{1}$ & $10^{2}$ \\
\hline
\end{tabular}

TABLE V. THE RATE OF CONVERGENCE OF MRE ON THE CIRCLE REPRESENTED EXACTLY AND BY A POLYGON ON VARIOUS ELEMENTS

\begin{tabular}{|l|l|l|}
\hline $\begin{array}{l}\text { Function } \\
\text { Approximation }\end{array}$ & Exact Geometry & Polygon Geometry \\
\hline Constant & $(0.2) \mathrm{n}^{-2}$ & $(4.7) \mathrm{n}^{-2}$ \\
\hline Hat & $(0.4) \mathrm{n}^{-2}$ & $(5.2) \mathrm{n}^{-2}$ \\
\hline Gauss & $(0.3) \mathrm{n}^{-3}$ & $(16.2) \mathrm{n}^{-2}$ \\
\hline
\end{tabular}

\section{DISCUSSION}

In problems 1 and 2 above, we were trying to recover the solution of equation (20) for the unit square and circle. For instance, with four 
elements, $\boldsymbol{u}$ was approximated with highest value of 11.5487 and 3.6252 for the square and circle respectively. For the square, there is a big absolute difference between the calculated and the exact $v$ of 9.8657. The circle had exact $v$ compared with the calculated one gave an absolute difference of only 4.2989. A better accuracy was realized when more elements on each side of the square or circle is increased. The programs Beint.m and Beintc.m were used for this analysis for the square and circle respectively. Noted also was that the maximum absolute errors in the approximation become smaller as you move from the left to the right of the boundary and reduce for increased $n$ (See tables 1 and 2 above; Figure 4) [2].

Regarding problem 3 , we majorly minded the domain that the circle or polygon enclosed as analyzed in the tables 3,4 , and 5 . The behavior of the mre reflects proportionality to $n^{-2}$. If for example if $n$ $=128$, the constant proportionality is 0.21 and therefore the error is approximated by $0.2\left(n^{-2}\right)$. If the geometry is exactly represented, the Gauss elements (do not represent a continuous approximation) give a higher order of convergence which is $o\left(n^{-3}\right)$. The hat and constants elements give slightly the same order of convergence $o\left(n^{-2}\right)$. On the contrary, the polygon geometry gave an order of convergence for the Gauss elements of $o\left(n^{-2}\right)$ [10], [11].

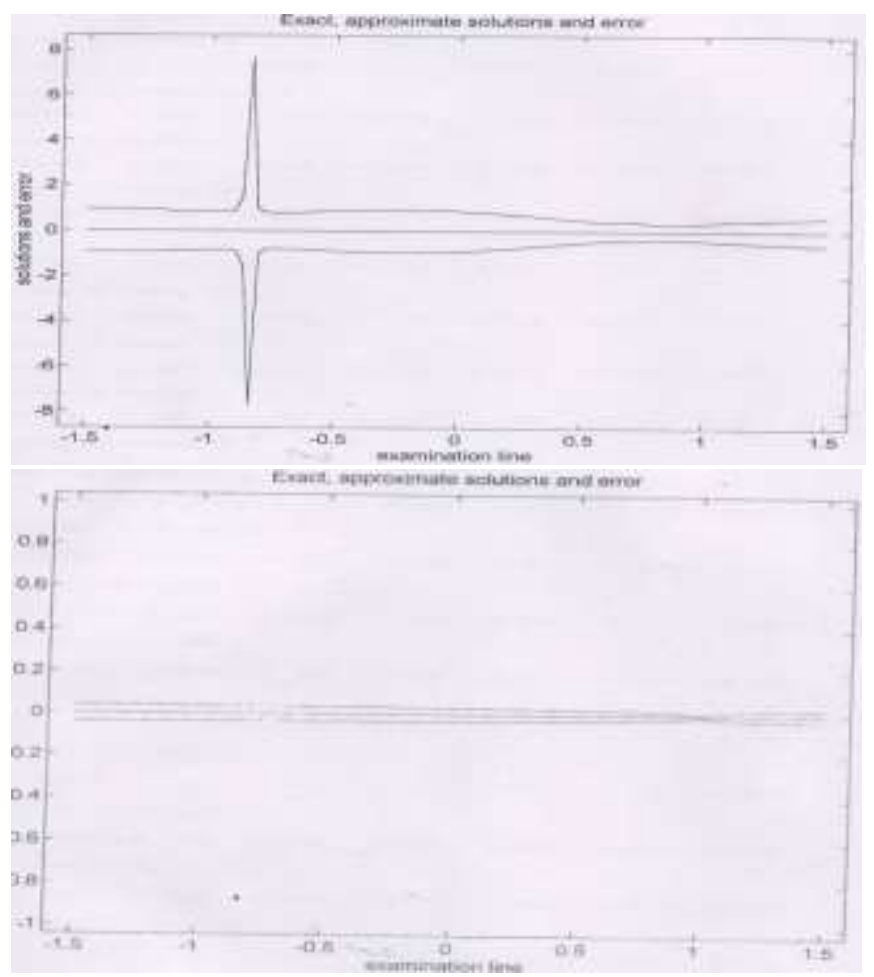

Figure. 4. Beintc. $m$ results for $n=8$ and $n=64$ [2]

Similarly, when straight elements are applied to such cases, they are either too much of approximations or they completely fail to work. For example, unless we use the curved elements, it becomes very difficult to establish the charge on the elliptic problem as the problem above.

\section{ACKNOWLEDGMENTS}

This work was made possible through research funding by Busitema University, Tororo, Uganda, where am employed.

\section{REFERENCES}

[1] Wrobel , L.C. \& Aliabad, M.H. (2002). The Boundary Element Methods in Engineering, McGraw-Hill College.
[2] Semwogerere, T. (2015). An Analysis of the Curved elements to a 2D Laplace Problem Using the BEM. Journal of Audio Engineering Mathematics and Physics, Vol. 5, No. 3, pp. 167-176.

[3] Rjasanow, S, \& Weggler, L. (2013). Accelerated High Order BEM for Maxwell Problems. Journal of Computational Mechanics, 51(431-441).

[4] Dennemeyer, R (1968). Introduction to Partial Differential Equations and Boundary Value Problems, Mc- Graw-Hill, Inc. USA, pp. 92-156.

[5] Zhu, S \& Satravaha, P \& Lu, X. (1994). Solving Linear Diffusion Equations with the Dual Reciprocity Method in Laplace Space. Journal of Engineering Analysis with Boundary Elements. 13(309-318).

[6] Henwood, D. J. (1997). The Boundary Element Method Applied to Laplace's Equation. Journal of Audio Engineering Society, Vol.6, pp. 1-19.

[7] Chen, G \& Zhou, J. (1992). Boundary Element Method, Academic Press Ltd: Harcourt Brace Jovanovich Publishers, London, pp. 1-16, 135-190, 374-403.

[8] Bremer, J \& Gimbutas, Z. (2012). A Nystrom Method for Weakly Singular Integral Operators on Surfaces. Journal of Computational Physics, 231(48854903).

[9] Fata, S. N. (2009). Explicit Expressions for 3D Boundary Integrals in Potential Theory. Journal of Numerical Methods in Engineering, 78(32-47).

[10] Henwood, D. J. \& Kirkup, S. M. (1994). An Empirical Analysis of the Boundary Element Method Applied to Laplace's Equation. Journal of Applied Mathematical Modeling, 2(32-36).

[11] Rashed Y.F., A coupled BEM-flexibility force method for bending analysis of internally supported plates. International Journal of Numerical Method in Engineering 2002, 54, 1431-1457.

\section{AUTHOR'S BIOGRAPHY}

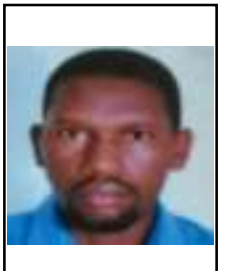

Mr. Twaibu Semwogerere was born in August, 1973, and holds a Bachelor of Science Education degree (1995) (Mathematics), Master of Science degree (2002) (Applied Mathematics) of Makerere University, Kampala, Uganda. $\mathrm{He}$ has a work experience of over 21 years in colleges and university teaching. Published several papers, the previous one being: "Exploration of Gravel Roads Erosion Data" published in CEET'2015 Conference Proceedings. He is currently a $\mathrm{Ph}$. D student (Engineering Mathematics) in the College of Engineering Design, Art, and Technology (CEDAT), Makerere University and a senior lecturer at Busitema University. His areas of research and teaching include Stochastic Processes, Probability Theory, and Numerical Analysis. 